\title{
Immobilized catalysts in synthesis of cyclic carbonates from $\mathrm{CO}_{2}$
}

\author{
$\underline{\text { Agnieszka Siewniak }}^{1}$ \\ 1. Department of Chemical Organic Technology and Petrochemisty, Silesian University of Technology, POLAND, \\ Gliwice, Krzywoustego 4, E-mail: Agnieszka.Siewniak@polsl.pl
}

\begin{abstract}
The possibility of obtaining cyclic alkylene carbonates from $\mathrm{CO}_{2}$ using immobilized catalysts in an one-pot synthesis was investigated. The synthesis proceeded in two stages: oxidation of olefin to epoxide and subsequent reaction of the obtained epoxide with $\mathrm{CO}_{2}$. The most favorable conditions for conducting of each stage were determined.
\end{abstract}

Keywords - cyclic carbonates, carbon dioxide, immobilized catalysts

\section{Introduction}

Cyclic carbonates have a wide range of applications, among others as plasticisers and solvents, as well as in the synthesis of fine chemicals and polymers [1]. The process of obtaining cyclic alkylene carbonates from $\mathrm{CO}_{2}$ can be carried out in two main ways: by the reaction of carbon dioxide with epoxides or alkenes [2]. Among the catalysts for these reactions the quaternary onium salts including ionic liquids deserve special attention because they are characterized by high activity.

The direct synthesis method using $\mathrm{CO}_{2}$ and olefin as substrates is becoming more and more popular. It runs in two stages (Fig. 1). The first one involves the epoxidation reaction of terminal olefins and in the second one in situ formed epoxide reacts with $\mathrm{CO}_{2}$. The advantage of this method is that both process steps can take place in one reaction vessel, so there is no need to isolate and purify the epoxide formed in the reaction. In addition relatively cheap and readily available reagents: alkenes and $\mathrm{CO}_{2}$, are used in this process. Currently, the key challenge for this technology is the development of new catalysts with high activity and selectivity that would allow the process to run under mild conditions. A particularly interesting aspect is the search for catalysts that can be easily separated after the reaction. This will allow to simplify usually costintensive separation operations of the post-reaction mixture.

\section{Results}

The paper presents studies on the synthesis of cyclic alkylene carbonates from $\mathrm{CO}_{2}$ and olefins using immobilized ionic liquids on insoluble carriers as catalysts. The tert-butyl hydroperoxide was used as an oxidant. The influence of selected reaction parameters: temperature, $\mathrm{CO}_{2}$ pressure, amount of catalyst, molar ratio of reagents on the course of each stage of the synthesis of cyclic carbonates was determined.

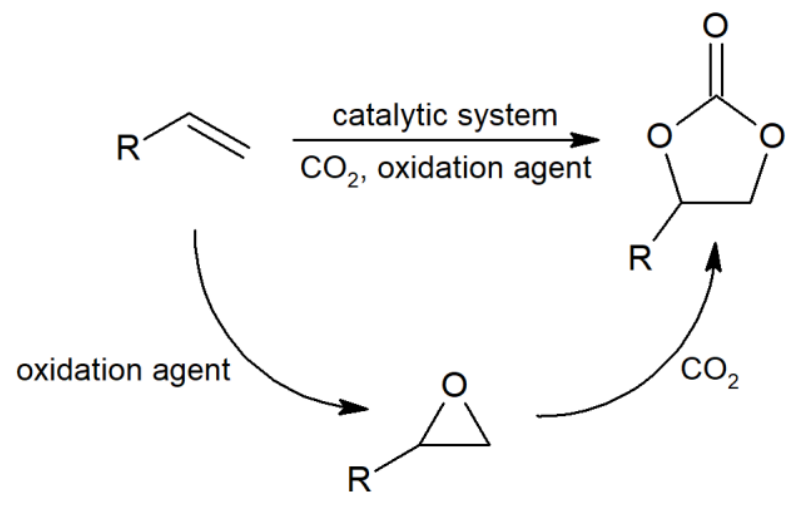

Fig.1. Scheme of synthesis of cyclic carbonates. 
The effect of types of inorganic carrier such as silica, as well as the addition cocatalysts, e.g. metal halides, on the activity of catalysts in the synthesis of cyclic carbonates was investigated. It is worth noting that the process did not need a solvent addition.

\section{Conclusion}

In this paper the catalysts based on ionic liquids immobilized on insoluble supports for the direct synthesis of cyclic carbonates was investigated. The use of such catalysts allowed easy separation of the post-reaction mixture, easy catalyst separation, e.g. by filtration, and enabled repeated use of the catalyst in the next processes.

\section{Acknowledgments}

This work was co-supported by National Science Centre (NCN), Poland, project No. 2018/02/X/ST8/03066: "Immobilized catalysts in the synthesis of cyclic carbonates from olefins and $\mathrm{CO}_{2}$ ".

\section{References}

[1] J. H. Clements, "Reactive Applications of Cyclic Alkylene Carbonates," Ind. Eng. Chem. Res., 42, 4, pp. 663-674, 2003.

[2] T. Sakakura, J. C. Choi, H. Yasuda, Chem., "Transformation of Carbon Dioxide," Chem. Rev., 107, 6, pp. 2365-2387, 2007. 